\title{
Superantigen Architecture: Functional Decoration on a Conserved Scaffold
}

\author{
Vickery L. Arcus and Edward N. Baker
}

\begin{abstract}
INTRODUCTION
A defining and consistent feature of the bacterial superantigens from Staphylococcus aureus and Streptococcus pyogenes is their strongly conserved three-dimensional structure. Structural studies to date show that the array of more than 280 amino acid sequences known for superantigens (SAgs) and staphylococcal superantigen-like (SSL) proteins all have the same fold-a structure in which the same three-dimensional arrangement of $\alpha$-helices and $\beta$-sheets is traced by each amino acid sequence, with the same topology (for recent reviews, see references 29 and 43). A typical SAg structure comprises two domains-an $\mathrm{N}$-terminal $\beta$-barrel domain called an $\mathrm{OB}$-fold $(4,25)$ and a $C$-terminal $\beta$-grasp domain in which a long $\alpha$-helix packs on to a mixed parallel and antiparallel $\beta$-sheet. These two domains are traversed by an $\alpha$-helix that lies at the $\mathrm{N}$ terminus of the protein and packs against the $\beta$-grasp domain, thus linking the $\mathrm{N}$ - and $\mathrm{C}$-terminal domains,

In striking juxtaposition with the conserved architecture among SAgs is the myriad of functions that have been grafted on to the SAg fold. For example, SAgs bind to major histocompatibility class II molecules (MHC-II), in some cases via an N-terminal binding face $(17,18)$, and in other cases via a C-terminal binding face $(24,30)$. In the case of staphylococcal enterotoxin A (SEA), both binding faces are utilized, resulting in simultaneous binding and cross-linking of MHC-II molecules at the antigen-presenting-cell surface (2, $16,44)$. Some SAgs mediate their binding to MHC-II via a zinc ion and some use their $\mathrm{N}$ terminal binding faces for oligomerization (38). SAgs also bind to T-cell receptors (TCRs), and although the TCR binding surface is consistent in its location on the SAg fold among different members of the SAg family, variation in sequence allows selectivity in binding to differing cohorts of TCRs.
\end{abstract}

Vickery L. Arcus - AgResearch Protein Engineering Laboratory, Department of Biological Sciences, University of Waikato, Private Bag 3105, Hamilton, and Centre for Molecular Biodiscovery. School of Biological Sciences, University of Auckland, Private Bag 92-019, Auckland, New Zealand. $\quad$ Edward N. Baker - Centre for Molecular Biodiscovery, School of Biological Sciences, University of Auckland, Private Bag 92-019, Auckland, New Zealand. 
Further variation has recently been seen for the staphylococcal superantigen-like proteins (SSLs) from the pathogenicity island SaInP2 $(19,47)$. which do not form complexes with the TCR and MHC-II molecules but instead interact with other components of the human immune system, apparently to dilute its ability to fight infection. Recently, members of this family have been shown to bind to immunoglobulin A (IgA) and complement C5 (20). Again, the conserved SAg fold has served as a scaffold for a diverse range of functions.

A logical extension of this theme is provided by the recently reported structures of the C5a receptor blocking domain (14) and the EAP domains (13), which are secreted staphylococcal virulence factors whose architecture comprises a single $\beta$-grasp domain. These proteins interact with the C5a receptor and extracellular host proteins, respectively. In a similar vein, the N-terminal SAg domain, the OB-fold, is well known as a "binding" domain capable of supporting an array of functions from binding to DNA, RNA, oligosaccharides, and proteins $(4,25)$.

An evolutionary story thus emerges for the SAg fold which suggests that this fold has arisen from the co-option of two versatile "binding" domains: the OB-fold and the $\beta$-grasp domain. These domains may then, through sequence variation, have adopted a wide range of binding modes to MHC-II molecules, the TCRs, and, in the case of the SSL proteins, a range of other components of the immune system. This is an evolutionary game of cat and mouse, as the toxicity of the SAgs would see them under heavy selection pressure to be eliminated by the immune system and, in return, the reciprocal pressure on SAgs is to vary widely and thus avoid the immune armory.

This chapter will first outline the details of the core three-dimensional structure of the SAgs and discuss precisely what is conserved among the family along with the evolutionary reasons for conservation. We will then discuss the variation that has been grafted onto the conserved structure, allowing different members of the family to bind to MHC-II and the TCR in many different configurations, with different affinities and, in the case of TCR binding, different specificities.

\section{THE SUPERANTIGEN FOLD: A CONSERVED SCAFFOLD}

The classic SAg fold is shown in Color Plate 3A using the structure of the streptococcal superantigen SMEZ-2 as an example (6). An N-terminal helix (at the top of the figure) packs into a groove between two structural domains. Following the $\mathrm{N}$-terminal helix is the $\mathrm{N}$ terminal domain, which is a five-stranded mixed $\beta$-barrel with Greek-key topology (48) and is called an OB-fold (25). This is connected to the C-terminal $\beta$-grasp domain by a large loop. The $\beta$-grasp domain is a mixed five-stranded $\beta$-sheet that packs onto a long $\alpha$ helix. Each domain has a hydrophobic core at its center, and a smaller hydrophobic core is formed by packing the $\mathrm{N}$-terminal helix onto one face of the $\beta$-grasp domain. In this sense, the folding of the SAg polypeptide chain for each domain, and for the protein as a whole, fits the classic paradigm for proteins, with a hydrophobic interior and a hydrophilic exterior. This also highlights and explains the conservation of residues along the central helix of the $\beta$-grasp domain, which constitutes the most highly conserved SAg sequence motif and defines this protein family (Color Plate 3, B and C). Most of these conserved residues are hydrophilic but are buried among hydrophobic residues as a result of the packing of the three major structural elements-the $N$-terminal helix, the OB-fold, and the $\beta$-grasp domain. Hydrophilic residues that form polar interactions (hydrogen bonding or charge- 
charge pairing) in a buried hydrophobic environment are subject to tight evolutionary constraints. This is due to the large free-energy penalty that is paid should one residue of the pair be mutated in the course of evolution. If one residue of a pair that forms a buried polar interaction is lost, this leaves an unpaired charge or polar group in a hydrophobic environment and is energetically costly for the structure as a whole. This is in contrast to buried hydrophobic residues that, in most cases, can tolerate mutation to other hydrophobic residues with little energetic cost. Thus, the majority of residues that are highly conserved across the SAg family are buried polar or charged residues and their conservation reflects structural and not functional constraints.

The SAg N-terminal domain forms a $\beta$-barrel with the OB-fold topology, a fold that is ubiquitous in nature; OB-fold domains are found in archaea, bacteria, and eukaryotes and are one of the most prevalent structural architectures (35). The OB-fold was first named by Murzin as an "oligosaccharide/oligonucleotide binding" fold when he noted that different examples of this fold could bind different ligands on the same face of the protein (25). This led to the suggestion that the OB-fold architecture can support a binding face that is easily adapted to the binding of a range of different ligands. This original observation, based on just four structures, has been borne out by the more than $90 \mathrm{OB}$-fold structures that have been deposited in the Protein Data Bank (PDB). Among these structures are OB-fold domains that use their binding face to bind RNA (the anticodon binding domains of the Aspand Lys-tRNA synthetases), single-stranded DNA (the telomere-end-binding proteins of eukaryotes and phage-derived gene $\mathrm{V}$ proteins), double-stranded DNA (cold-shock proteins), and oligosaccharides (bacterial $A_{5}$ toxins). The $O B$-fold-binding face has even been adapted for catalysis in the inorganic pyrophosphatases (4).

The SAgs and SAg-like proteins use the binding face of their OB-fold domain as a protein-protein interaction domain. Even for this protein family, there is wide functional variation. A group of SAgs including SEB, SEC1-3, SSA, and SPE-A use the OB-fold binding face to bind to the $\alpha$-chain of MHC-II $(17,28,40)$. TSST- 1 and TSST- 2 use the same face to bind to the $\alpha$-chain of MHC-II, but in a different orientation (18). In contrast, SPE-C uses this OB-fold binding face to oligomerize and form dimers (38). The OB-fold is sufficiently tolerant to mutation that there are just two short sequence motifs which define the SAg N-terminal OB-fold domain. These residues are buried at the interface between the two SAg domains and are conserved for structural rather than functional reasons.

The superantigen $\mathrm{C}$-terminal $\beta$-grasp domain also presents a binding face at the surface of its mixed $\beta$-sheet. This binding face is used by, for example, SEA, SPE-C, SMEZ-2, SPE-G, SPE-J, and SEH to bind to the $\beta$-chain of MHC-II molecules $(24,26,30,33)$. The binding is zinc dependent; a zinc ion is ligated by two histidine side chains and an aspartic acid at this face. The recent three-dimensional structure of SPE-C in complex with MHCII shows this interaction in detail (24). Upon complex formation, tetrahedral coordination of the $\mathrm{Zn}^{2+}$ ion is completed by His- 81 from the $\beta$-chain of MHC-Il.

The $\beta$-grasp domain is also very well represented in nature. Many examples of this architecture are intriguing in the light of SAg structure and activity. The $\beta$-grasp domains from streptococcal immunoglobulin-binding protein interact with IgG antibodies to subvert the immune response (10). The thrombolytic agents staphylokinase and streptokinase are also $\beta$-grasp protein-protein binding domains $(36,46)$. More recently, two other virulence factors from Streptococcus and Staphylococcus have been found to contain $\beta$-grasp domains with structural homology to the SAg C-terminal domain. The first of these is the 
extracellular adherence protein (EAP) domain from S. aureus (13). EAP is secreted by the bacteria and is thought to aid pathogenicity by interacting with host proteins such as fibrinogen, fibronectin, and vitronectin, which, in turn, leads to agglutination. The full-length protein is between 50 and $70 \mathrm{kDa}$ and contains, in different bacterial strains, either four or six $\beta$-grasp domains. Notably, the EAP $\beta$-grasp domains have no recognizable sequence homology with SAg C-terminal domains (13).

The C5a receptor blocking domain of the chemotaxis inhibitory protein from $S$. aureus is also a pathogenicity factor and also a $\beta$-grasp domain (14). In this case, the secreted protein binds to the $C 5$ a receptor through its $\beta$-grasp domain and interferes with phagocyte responses.

The affinity for a range of ligands shown by these other $\beta$-grasp domains is consistent with our paradigm for SAgs, whereby adaptable binding scaffolds are utilized by secreted proteins in Staphylococcus and Streptococcus pathogenesis.

\section{ALLELIC VARIATIONS IN SEQUENCE DECORATE THE SUPERANTIGEN FOLD}

The variation among $S A g$ sequences is a continuum from allelic variation where sequences from different strains differ by just a few amino acids ( $>98 \%$ identity), to pairwise sequence identities of less than $10 \%$. In the case of allelic variation, the differences are almost universally found at the surface of the proteins and suggest that these are occurring under selection to vary epitopes that are targeted by the immune system. For example, the extraordinarily potent streptococcal superantigen, SMEZ, has 21 alleles that segregate with different M/emm types (34). Of the 31 amino acid positions that vary across the SMEZ sequence, 26 of these amino acids are surface exposed. Color Plate 3D maps these positions onto the structure of SMEZ-2. Similarly, five alleles of the SEC superantigen have been reported, including one from a strain of $S$. aureus isolated from a bovine infection. Once again, with just one exception, the variations are seen at the protein surface.

A similar phenomenon is seen for the SSL family of proteins, which share the SAg fold but bind other components of the immune response, and which are encoded on a pathogenicity island of $S$. aureus $(19,47)$. These 11 genes lie adjacent to one another and upstream from a putative transposase gene, suggesting a capacity for this island to be horizontally transferred. A survey of different $S$. aureus strains showed that all strains carry the pathogenicity island and that a subset of strains carries all 11 genes on the island; in other strains, up to four SSL genes have been deleted from the pathogenicity island (12). Allelic variation is seen for each of the SSL proteins ( 85 to $100 \%$ identity) and can be mapped on to the three-dimensional structures of the two SSLs for which structures are available, SSL-5 and SSL-7 $(5,20)$ (M. Chung, personal communication). The same theme of surface variation is continued for this family of immune interactors, such that in the case of SSL-5, 32 of the 37 positions that vary between alleles lie at the surface of the protein and the remaining 5 variants are buried hydrophobic residues (5).

The mosaic nature of the allelic variants for both SMEZ and SSL-5 has been cited as evidence that these alleles arise principally from recombination and not from point mutation (34). However, it is likely that a combination of both effects applies. Although the origins of this variation remain equivocal, the outcome is compelling-wide allelic variation at the surface of many SAgs and SSLs is most probably driven by the constant evolutionary pressure to escape host immune detection. 


\section{VARIATIONS ON SUPERANTIGEN BINDING TO MHC-II AND TCR}

At one end of the spectrum of SAg variability lie the allelic variants (discussed above), which presumably retain the same functional determinants within a particular molecule. At the other end of the spectrum are SAgs that have not only disparate sequences but also altered functional modes of action. At the extreme end of this spectrum lie the SSLs, EAP, C5a, and IgG-binding proteins, all of which share clear structural relationships with the SAgs but target other protein components of the immune response.

The interactions between SAgs and their target MHC-II molecules and TCRs cover virtually all permutations of the following binding modes: MHC-II $\alpha$-chain binding; MHC-II $\beta$-chain binding; TCR V $\alpha$ binding and restriction; TCR V $\beta$ binding and restriction; SAg oligomerization; MHC-II cross-linking. For the purposes of discussion we can loosely divide these variations into MHC-II $\alpha$-chain binding and its associated TCR interactions, MHC-II $\beta$-chain binding and its associated TCR interactions, and SAg oligomerization and MHC-II cross-linking.

\section{MHC-II $\alpha$-Chain Binding}

SAgs that bind to the $\alpha$-chain of MHC-II molecules generally do so with low affinity (in the range 0.1 to $1.0 \mu \mathrm{M}$ ) (37). There are several three-dimensional structures of SAg/MHC-II complexes that show this interaction in molecular detail, epitomized by the SEB/MHC-II complex structure (see Color Plate 4A) (17). Loops that lie above and below the binding face of the OB-fold domain of SEB form both polar and hydrophobic interactions with residues from the MHC-II $\alpha$-chain helix that flanks the peptide-binding groove. In addition, interactions are made between SEB and loops at the end of the $\beta$-sheet that forms the floor of the MHC-II peptide-binding groove. The small surface area that is buried on complex formation between SEB and MHC $\left(660 \AA^{2}\right)$ accounts in part for the low affinity of this interaction.

This SAg/MHC-II binding interface is conserved when the structures of the MHC-II complexes with SEB and SEC 3 are compared $(17,42)$. Just two conservative mutations are at the MHC-II-binding interface when SEB is compared with SEC3, despite an overall sequence identity of $66 \%$ between the two SAgs. Other SAgs from both S. aureus and S. pyogenes that interact with MHC-II in this manner are SEC1, SEC2, SEG, SSA, and SPE-A.

The binding of this group of SAgs to the TCR has also been well defined by the experimentally determined structures of SEB, SEC2, SEC3, and SPE-A, each in complex with mouse TCR V $\beta 8.2(11,22,41)$. Residues on the SAgs that are involved in binding lie on the face between the two domains and at the surface of the $\mathrm{N}$-terminal $\alpha$-helix. The regions involved on the TCR are primarily located on the CDR2 and HV4 loops and the adjacent FR3 regions (see Color Plate 5). From Color Plate 5A, it is evident that this is a weak proteinprotein interaction with just three long hydrogen bonds between side chains of SEB and main-chain atoms of the TCR. Additionally, the buried interface is relatively small at $540 \AA^{2}$.

The inferred functional complex for MHC-I/SEB/TCR can be constructed from the experimentally determined MHC-II/SEB and SPE-A/TCR binary complexes. This presents a picture of a circular, ternary complex where each of the components interacts with the other (Color Plate 6). Here, the SAg interferes with the normal MHC-II/TCR interaction by inserting the $\mathrm{N}$-terminal OB-fold domain between the MHC-II $\alpha$-chain and the TCR $\beta$-chain. The TCR now binds to both the SAg and the $\beta$-chain of MHC-II. It has been speculated that the cooperative set of interactions that form between TCR, MHC-II, 
and SAg in the complete complex may compensate for the low affinities that are seen in complexes between the pairs of protagonists $(3,37)$.

TSST is an enigma among MHC-II $\alpha$-chain-binding SAgs. TSST binds to MHC-II in a different orientation when compared with SEB/MHC-II binding, lying across the $\alpha$-helix that flanks the peptide-binding groove and making contacts with the displayed peptide (18, 45). It is not known what implications this alternative SAg/MHC-II interaction would have on the TSST/MHC-II/TCR complex, and no direct structural information is available on the mode of TSST/TCR interaction.

\section{MHC-II $\beta$-Chain Binding}

A second group of SAgs bind to the $\beta$-chain of MHC-II. The SAgs in this group (SPE-C, SPE-J, SMEZ, SED, SEH, and SEJ) are illustrated by SPE-C and SEH, for which the structures of SAg/MHC-II complexes have recently been reported $(24,30)$. At the center of the binding interface is a zinc ion that is tetrahedrally coordinated by three ligands from the $\mathrm{SAg}$ and a single histidine from MHC $\beta$-chain (His 81 , see Color Plate $4 \mathrm{~B}$ ). The $\mathrm{SAg} / \mathrm{MHC}$-II binding has been shown to be zinc dependent in vivo, such that addition of the zinc-chelating agent EDTA abolishes T-cell activation $(23,33)$. The SAg interface covers residues on the $\mathrm{MHC}$-II $\beta$-chain helix along with residues of the displayed peptide. In contrast to the binding between SEB and the $\alpha$-chain of MHC-II, the affinity of the interaction between the SPE-C group SAgs and the $\beta$-chain of MHC is some three orders of magnitude greater $(0.1$ to $100 \mathrm{nM})(23,26,33)$. A comparison of the surface area buried by SEB binding to the $\alpha$-chain of MHC-II with that buried by SPE-C binding to the $\beta$-chain of MHC-II shows that the surface areas are similar, and by implication, the binding affinities should also be comparable. However, the bonding that results from the involvement of a transition metal (zinc) bound with optimal tetrahedral geometry at the interface confers tighter binding on the SPE-C/MHC-Il $\beta$-chain interaction.

This orientation of SPE-C in binding to the $\beta$-chain of MHC-II dictates that the TCR now binds to SPE-C at a distance from MHC-II, producing a more linear MHCII/SAg/TCR complex (Color Plates 5B and 7A) (41). Although the TCR binding site on SPE-C is in the same region as that on SEB, some important differences exist in the detail of the SAg/TCR interactions. SPE-C has more extensive interactions with the TCR including the CDR1, CDR2, and CDR3 loops of the $\beta$-chain of the TCR, along with the HV4/FR3 components (41). The SPE-C/TCR interface also includes 9 hydrogen bonds that are in some cases mediated by side chains from both the SAg and TCR. Whereas the SEB interactions primarily involve hydrogen bonds with main-chain atoms of the TCR loops, and thus primarily discriminate between TCRs on the basis of the backbone conformations of these loops (22), the SPE-C interaction is highly specific. This is due to the more extensive range of interactions between SAg and TCR, and to the unique conformations of CDR loops of human VB2.1 due to amino acid insertions on CDR1 and CDR2 loops and an extended CDR3 loop (see Color Plate 5B). The affinity of the SPE-C/TCR interaction is also greater than that seen between SEB and TCR (21) and this is reflected in a significantly greater burial of surface area on complexation $\left(810 \AA^{2}\right)$.

The increased affinity of both the SPE-C/MHC-II and the SPE-C/TCR interactions may be required due to the linear configuration of the ternary complex, which precludes cooperativity of binding between the three components. The hypothesis is that stimulation of 
the T cells requires long residence times for the SAg to cross-connect TCR and MHC-II, and this can be achieved either by independent binding interactions of nanomolar affinity, in the case of the linear MHC-II/SPE-C/TCR complex, or by triangular, cooperative, binding to achieve nanomolar affinity in the circular MHC-II/SEB/TCR complex.

In the case of SEH, there is evidence for TCR V $\alpha$ chain selectivity, although it is not clear how this is effected. It has been reported that there is upregulation of human TCR V $\alpha 10$ T cells after SEH stimulation (31). SEH bridges the linear MHC-IU/SAg/TCR complex and it is possible that the SEH/TCR interaction may include binding to the TCR V $\alpha$ region (31). In addition a single report has demonstrated a role for TCR V $\alpha$ interactions with SEA and SEE in the T-cell response to SAgs (9).

\section{Cross-Linking MHC-II}

Two SAgs have been demonstrated to cross-link MHC-II molecules at the antigen-presenting cell (APC) surface. SEA achieves this by simultaneously binding to the $\alpha$-chain of MHC-II in an SEB-like manner (using the OB-fold binding face), and to the $\beta$-chain of $\mathrm{MHC}$-II in a SPE-C-like manner (using the $\beta$-grasp binding face at the opposite end of the molecule) $(1,16,32)$. The resulting complex at the APC/T-cell interface has a stoichiometry of $\mathrm{MHC}_{2} / \mathrm{SEA} / \mathrm{TCR}$. Note that an MHC-II/SEA $\mathrm{A}_{2}$ complex could not bind to the TCR, as simultaneous binding of two SEA molecules to a single MHC at both $\alpha$ - and $\beta$-chains would occlude TCR binding in a triangular MHC-II/SEA/TCR complex (32). It has been proposed that rafting of MHC-II molecules at the cell surface facilitates the release of cytokines from the APC and that cross-linking by SEA may contribute to the overproduction of cytokines that is characteristic of toxic shock (16). By sequence homology, it is proposed that SED and SEE also cross-link MHC-II in a similar fashion.

A second variation is the cross-linking of MHC-II that can be effected by SAg dimers. Thus, SPE-C has been shown to form dimers through its OB-fold domain $(23,38)$, leaving the C-terminal $\beta$-grasp domain on each SPE-C monomer free to make its $\mathrm{Zn}$-mediated interaction with MHC-II (24). This results in a proposed complex of the type (MHC/SPE$\mathrm{C} / \mathrm{TCR})_{2}$ (Color Plate 7B). SPE-C is a dimer both in solution and in the crystal structure, and the importance of the dimer for T-cell stimulation has been demonstrated, although this remains controversial. A second form of dimerization is shown by the streptococcal SAg SPE-J (7). In this case, the dimerization surface overlaps the TCR binding surface, implying that dimerization and TCR binding are mutually exclusive, but that SPE-J dimers could still function in MHC-II cross-linking.

\section{SAg Oligomerization}

The question of SAg oligomerization is controversial. Most SAgs appear to act as monomers, but some, such as SPE-C and SPE-J (discussed above), can form dimers, albeit at relatively high concentrations. Crystal structures of other SAgs, such as TSST-1 (27) and SPE-A (8), have also suggested oligomeric associations whose significance is unclear; given that these associations must be weak, their relevance in vivo will depend on local environments and local concentrations. An instructive example is provided by the SAg-like protein SSL-5 (formerly known as SET3). The dimer found in crystals of SSL-5 buries only a small surface and is not detected in solution (5). It may still be biologically relevant, however, where its binding partner(s) provide added stabilization, a suggestion given 
weight by the observation that a similar mode of dimerization is found for SSL-11 $(\mathrm{H}$. Baker and $\mathrm{M}$. Chung, manuscript in preparation).

\section{CONCLUDING REMARKS}

As secreted proteins from two highly adapted human pathogens, S. aureus and S. pyogenes, SAgs and the related SSLs must be subject to severe immune pressure due to their potent effects. This serves as an explanation for their defining structural characteristicextensive surface sequence variability superimposed onto a highly conserved fold that is built from two promiscuous binding modules (the OB-fold and $\beta$-grasp domains). The result is a diversity of binding properties, in which many protein-protein interactions are weak. The challenge is to understand which interactions are of real physiological significance and which are not; only when cooperativity of binding occurs or specific features arise, such as the zinc site on the $\beta$-grasp domain, are high-affinity complexes formed, but other, more transient, associations may still be relevant in vivo. The implications for infection and disease are similar. Despite their notoriety, it is likely that, for the most part, the effects of SAg and SSL secretion are relatively benign, and that only when local concentrations rise, or synergistic relationships with other factors apply, do they trigger the severe invasive disease with which they are associated.

Acknowledgments. We gratefully acknowledge the Health Research Council of New Zealand for its support of our research on superantigen structure, John Fraser and Thomas Proft for many stimulating discussions, and Matthew Chung and Heather Baker for access to unpublished data.

\section{REFERENCES}

1. Abrahmsen, L., M. Dohlsten, S. Segren, P. Bjork, E. Jonsson, and T. Kalland. 1995. Characterization of two distinct MHC class II binding sites in the superantigen staphylococcal enterotoxin A. EMBO J. 14:29782986.

2. Al-Daccak, R., K. Mehindate, F. Damdoumi, P. Etongue-Mayer, H. Nilsson, P. Antonsson, M. Sundstrom, M. Dohlsten, R. P. Sekaly, and W. Mourad. 1998. Staphylococcal enterotoxin D is a promiscuous superantigen offering multiple modes of interactions with the MHC class II receptors. J. Immunol. 160:225-232.

3. Andersen, P. S., P. M. Lavoie, R. P. Sekaly, H. Churchill, D. M. Kranz, P. M. Schlievert, K. Karjalainen, and R. A. Mariuzza. 1999. Role of the T cell receptor alpha chain in stabilizing TCR-superantigen-MHC class II complexes. Immunity 10:473-483.

4. Arcus, V. L. 2002. OB-fold domains: a snapshot of the evolution of sequence, structure and function. Curr. Opin. Struct. Biol. 12:794-801.

5. Arcus, V. L., R. Langley, T. Proft, J. D. Fraser, and E. N. Baker. 2002. The three-dimensional structure of a superantigen-like protein, SET3, from a pathogenicity island of the Staphylococcus aureus genome. J. Biol. Chem. 277:32274-32281.

6. Arcus, V. L., T. Proft, J. A. Sigrell, H. M. Baker, J. D. Fraser, and E. N. Baker. 2000. Conservation and variation in superantigen structure and activity highlighted by the three-dimensional structures of two new superantigens from Streptococcus pyogenes. J. Mol. Biol. 299:157-168.

7. Baker, H. M., T. Proft, P. D. Webb, V. L. Arcus, J. D. Fraser, and E. N. Baker. 2004. Crystallographic and mutational data show that the streptococcal pyrogenic exotoxin $J$ can use a common binding surface for $T$-cell receptor binding and dimerization. J. Biol. Chem. 279:3857l-38576.

8. Baker, M. D., I. Gendlina, C. M. Collins, and K. R. Acharya. 2004. Crystal structure of a dimeric form of streptococcal pyrogenic exotoxin A (SpeA1). Protein Sci. 13:2285-2290.

9. deAlba, Y. B., P. N. Marche, P. A. Cazenave, I. Cloutier, R. P. Sekaly, and J. Thibodeau. 1997. V alpha domain modulates the multiple topologies of mouse $T$ cell receptor $V$ beta $20 /$ staphylococcal enterotoxins $A$ and E complexes. Eur. J. Immunol. 27:92-99. 
10. Derrick, J. P., and D. B. Wigley. 1994. The 3rd lgG-binding domain from Streptococcal protein-G-an analysis by X-ray crystallography of the structure alone and in a complex with Fab. J. Mol. Biol. 243:906918.

11. Fields, B. A., E. L. Malchiodi, H. M. Li, X. Ysern, C. V. Stauffacher, P. M. Schlievert, K. Karjalainen, and R. A. Mariuzza. 1996. Crystal structure of a T-cell receptor beta-chain complexed with a superantigen. Nature 384:188-192.

12. Fitzgerald, J. R., S. D. Reid, E. Ruotsalainen, T. J. Tripp, M. Y. Liu, R. Cole, P. Kuusela, P. M. Schlievert, A. Jarvinen, and J. M. Musser. 2003. Genome diversification in Staphylococcus aluretus: molecular evolution of a highly variable chromosomal region encoding the staphylococcal exotoxin-like family of proteins. Infect. Immun. 71:2827-2838.

13. Geisbrecht, B. V., B. Y. Hamaoka, B. Perman, A. Zemla, and D. J. Leahy. 2005. The crystal structures of EAP domains from Staphylococcus aureus reveal an unexpected homology to bacterial superantigens. FASEB J. 19:A313-A314.

14. Haas, P. J., C. J. C. de Haas, M. Poppelier, K. P. M. van Kessel, J. A. G. van Strijp, K. Dijkstra, R. M. Scheek, H. Fan, J. A. W. Kruijtzer, R. M. J. Liskamp, and J. Kemmink. 2005. The structure of the C5a receptor-blocking domain of chemotaxis inhibitory protein of Staphylococcus aureus is related to a group of immune evasive molecules. J. Mol. Biol. 353:859-872.

15. Hennecke, J., and D. C. Wiley. 2002. Structure of a complex of the human alpha/beta T cell receptor (TCR) HA 1.7, influenza hemagglutinin peptide, and major histocompatibility complex class II molecule. HLA-DR4 (DRA*0101 and DRB1 *0401): Insight into TCR cross-restriction and alloreactivity. J. Exp. Med. 195:571-581.

16. Hudson, K. R., R. E. Tiedemann, R. G. Urban, S. C. Lowe, J. L. Strominger, and J. D. Fraser. 1995. Staphylococcal-enterotoxin-A has 2 cooperative binding-sites on major histocompatibility complex class-II. J. Exp. Med. 182:711-720.

17. Jardetzky, T. S., J. H. Brown, J. C. Gorga, L. J. Stern, R. G. Urban, Y. I. Chi, C. Stauffacher, J. L. Strominger, and D. C. Wiley. 1994. 3-Dimensional structure of a human class-Il histocompatibility molecule complexed with superantigen. Nature 368:711-718.

18. Kim, J. S., R. G. Urban, J. L. Strominger, and D. C. Wiley. 1994. Toxic shock syndrome toxin-1 complexed with a class-II major histocompatibility molecule HLA-DR1. Science 266:1870-1874.

19. Kuroda, M., T. Ohta, I. Uchiyama, T. Baba, H. Yuzawa, I. Kobayashi, L. Cui, A. Oguchi, K. Aoki, Y. Nagai, J. Lian, T. Ito, M. Kanamori, H. Matsumaru, A. Maruyama, H. Murakami, A. Hosoyama, Y. Mizutani-Ui, N. K. Takahashi, T. Sawano, R. Inoue, C. Kaito, K. Sekimizu, H. Hirakawa, S. Kuhara, S. Goto, J. Yabuzaki, M. Kanehisa, A. Yamashita, K. Oshima, K. Furuya, C. Yoshino, T. Shiba, M. Hattori, N. Ogasawara, H. Hayashi, and K. Hiramatsu. 2001. Whole genome sequencing of meticilinresistant Staphylococcus aureus. Lancet 357:1225-1240.

20. Langley, R., B. Wines, N. Willoughby, I. Basu, T. Proft, and J. D. Fraser. 2005. The staphylococcal superantigen-like protein 7 binds $\operatorname{IgA}$ and complement $\mathrm{C} 5$ and inhibits IgA-Fc alpha RI binding and serum killing of bacteria. J. Immunol. 174:2926-2933.

21. Leder, L., A. Llera, P. M. Lavoie, M. I. Lebedeva, H. M. Li, R. P. Sekaly, G. A. Bohach, P. J. Gahr, P. M. Schlievert, K. Karjalainen, and R. A. Mariuzza. 1998. A mutational analysis of the binding of staphylococcal enterotoxins B and C3 to the T cell receptor beta chain and major histocompatibility complex class II J. Exp. Med. 187:823-833.

22. Li, H. M., A. Llera, D. Tsuchiya, L. Leder, X. Ysern, P. M. Schlievert, K. Karjalainen, and R. A. Mariuzza. 1998. Three-dimensional structure of the complex between a $\mathrm{T}$ cell receptor beta chain and the superantigen staphylococcal enterotoxin B. Immunity 9:807-816.

23. Li, P. L., R. E. Tiedemann, S. L. Moffat, and J. D. Fraser. 1997. The superantigen streptococcal pyrogenic exotoxin C (SPE-C) exhibits a novel mode of action. J. Exp. Med. 186:375-383.

24. Li, Y. L., H. M. Li, N. Dimasi, J. K. McCormick, R. Martin, P. Schuck, P. M. Schlievert, and R. A. Mariuzza. 2001. Crystal structure of a superantigen bound to the high-affinity, zinc-dependent site on MHC class II. Immunity 14:93-103.

25. Murzin, A. G. 1993. OB (Oligonucleotide Oligosaccharide Binding)-fold-common structural and functional solution for nonhomologous sequences. EMBO J. 12:861-867.

26. Nilsson, H., P. Bjork, M. Dohlsten, and P. Antonsson. 1999. Staphylococcal enterotoxin H displays unique MHC class II-binding properties. J. Immunol. 163:6686-6693.

27. Papageorgiou, A. C., R. D. Brehm, D. D. Leonidas, H. S. Tranter, and K. R. Acharya. 1996. The refined erystal structure of toxic shock syndrome toxin-1 at 2.07 angstrom resolution. J. Mol. Biol. 260:553-569. 
28. Papageorgiou, A. C., C. M. Collins, D. M. Gutman, J. B. Kline, S. M. O'Brien, H. S. Tranter, and K. R. Acharya. 1999. Structural basis for the recognition of superantigen streptococcal pyrogenic exotoxin A (SpeAl) by MHC class II molecules and T-cell receptors. EMBO J. 18:9-21.

29. Petersson, K., G. Forsberg, and B. Walse. 2004. Interplay between superantigens and immunoreceptors. Scand. J. Immunol. 59:345-355.

30. Petersson, K., M. Hakansson, H. Nilsson, G. Forsberg, L. A. Svensson, A. Liljas, and B. Walse. 2001 Crystal structure of a superantigen bound to MHC class II displays zinc and peptide dependence. EMBO J. 20:3306-3312

31. Petersson, K., H. Pettersson, N. J. Skartved, B. Walse, and G. Forsberg. 2003. Staphylococcal enterotoxin $\mathrm{H}$ induces $\mathrm{V}$ alpha-specific expansion of T cells. J. Immunol. 170:4148-4154.

32. Petersson, K., M. Thunnissen, G. Forsberg, and B. Walse. 2002. Crystal structure of a SEA variant in complex with MHC class II reveals the ability of SEA to crosslink MHC molecules. Structure 10:1619-1626.

33. Proft, T., S. L. Moffatt, C. J. Berkahn, and J. D. Fraser. 1999. Identification and characterization of novel superantigens from Streprococcus pyogenes. J. Exp. Med. 189:89-101.

34. Proft, 'T., S. L. Moffatt, K. D. Weller, A. Paterson, D. Martin, and J. D. Fraser. 2000. The streptococcal superantigen SMEZ exhibits wide allelic variation, mosaic structure, and significant antigenic variation. J. Exp. Med. 191:1765-1776.

35. Qian, J., B. Stenger, C. A. Wilson, J. Lin, R. Jansen, S. A. Teichmann, J. Park, W. G. Krebs, H. Y. Yu, V. Alexandrov, N. Echols, and M. Gerstein. 2001. PartsList: a web-based system for dynamically ranking protein folds based on disparate attributes, including whole-genome expression and interaction information. Nucleic Acids Res. 29:1750-1764.

36. Rabijns, A., H. L. DeBondt, and C. DeRanter. 1997. Three-dimensional structure of staphylokinase, a plasminogen activator with therapeutic potential. Nat. Struct. Biol. 4:357-360.

37. Redpath, S., S. M. Alam, C. M. Lin, A. M. O'Rourke, and N. R. J. Gascoigne. 1999. Cutting edge: Trimolecular interaction of TCR with MHC class II and bacterial superantigen shows a similar affinity to MHC:peptide ligands. J. Immunol. 163:6-10.

38. Roussel, A., B. F. Anderson, H. M. Baker, J. D. Fraser, and E. N. Baker. 1997. Crystal structure of the streptococcal superantigen SPE-C: dimerization and zinc binding suggest a novel mode of interaction with MHC class II molecules. Nat. Struct. Biol. 4:635-643.

39. Schuster-Boeckler, B., J. Schultz, and S. Rahmann. 2004. HMM logos for visualization of protein families. BMC Bioinformatics 5:7.

40. Sundberg, E., and T. S. Jardetzky. 1999. Structural basis for HLA-DQ binding by the streptococcal superantigen SSA. Nat. Struct. Biol. 6:123-129.

41. Sundberg, E. J., H. M. Li, A. S. Llera, J. K. McCormick, J. Tormo, P. M. Schlievert, K. Karjalainen, and R. A. Mariuzza. 2002. Structures of two streptococcal superantigens bound to TCR beta chains reveal diversity in the architecture of T cell signaling complexes. Structure 10:687-699.

42. Sundberg, E. J., P. S. Andersen, P. M. Schlievert, K. Karjalainen, and R. A. Mariuzza. 2003. Structural, energetic, and functional analysis of a protein-protein interface at distinct stages of affinity maturation. Structure 11:1151-1161.

43. Sundberg, E. J., Y, L. Li, and R. A. Mariuzza. 2002. So many ways of getting in the way: diversity in the molecular architecture of superantigen-dependent T-cell signaling complexes. Curr. Opin. Immunol. 14:36-44.

44. Tiedemann, R. E., and J. D. Fraser. 1996. Cross-linking of MHC class II molecules by staphylococcal enterotoxin A is essential for antigen-presenting cell and T cell activation. J. Immunol. 157:3958-3966.

45. Vonbonin, A., S. Ehrlich, G. Malcherek, and B. Fleischer. 1995. Major histocompatibility complex class Il-associated peptides determine the binding of the superantigen toxic shock syndrome toxin-1. Eur. J. Im munol. 25:2894-2898.

46. Wang, X. Q., X. L. Lin, J. A. Loy, J. Tang, and X. J. C. Zhang. 1998. Crystal structure of the catalytic domain of human plasmin complexed with streptokinase. Science 281:1662-1665.

47. Williams, R. J., J. M. Ward, B. Henderson, S. Poole, B. P. O'Hara, M. Wilson, and S. P. Nair. 2000. Identification of a novel gene cluster encoding staphylococcal exotoxin-like proteins: characterization of the prototypic gene and its protein product, SET1. Infect. Immun. 68:4407-1415.

48. Zhang, C., and S. H. Kim. 2000. A comprehensive analysis of the Greek key motifs in protein beta-barrels and beta-sandwiches. Proteins-Struct. Func. Genet. 40:409-419. 